\title{
Nursing Care Systems: Enhancing Care Processes in Practice and Management
}

\author{
H.F. Marin' ${ }^{1}$ R. Carr ${ }^{2}$ \\ 'Nursing Informatics Group, Federal University of São Paulo, São Paulo, Brazil \\ ${ }^{2}$ Informatics Project Contracting (IPC) \& Associates, Cambridge, Waikato, New Zealand
}

\begin{abstract}
Summary
Objective: To summarize aspects of nursing informatics application and introduces challenges that are currently being addressed by nurses around the world.

Methods: Key contributions in the field are surveyed and put in the perspective of the IMIA Nursing Informatics Strategic Plan.

Results: As a discipline, nursing informatics offers real and practical instruments and opportunities to delivery nursing care across settings, units of care and specialties. A variety of patient care systems are managed by nurses who are introduced to this link during their training embracing information networks, communication skills together with an ability to work both in practice and administrative positions in a variety of clinical and community settings.

Conclusion: At a time when the tendency is toward a computer-based patient record, nurses must be able to identify what kind of nursing data and information will construct useable and useful nursing knowledge. There are many options for each area of application and the selection must be done carefully, considering the enhancement for organizational, technological and local requirements.
\end{abstract}

\section{Keywords}

Nursing, informatics, information, communication, technology

Geissbuhler A, Kulikowski C, editiors. IMIA Yearbook of Medical Informatics 2008. Methods Inf Med 2008; 47 Supp 1 : 25-28

\section{Introduction}

Since information technology was included as a tool in the hospitals and healthcare facilities, the process of decision making, control and evaluation of nursing care has significantly altered. Currently, nursing informatics is an established and recognized area.

As a discipline, nursing informatics offers real and practical instruments and opportunities to deliver nursing care across settings, units of care and specialties. Nursing, along with other health providers, is an information dependable profession. Nurses together with all other health care professionals depend on accurate and timely access to appropriate information in order to perform the variety of activities that involve patient care, including administration, consulting, education, training and research. The information required is not only about technical knowledge, scientific development, quality control, clinical and administrative documentation but also about human and material resources available to deliver nursing care.

The decision for planning and delivering nursing care is always based on the information available from resources, science and technological development, patient needs and technical skills. The more specific information to support clinical decisions, the better the care that can be delivered to the patient/client. Furthermore, the quality of care is re- lated to the scope of knowledge and information that healthcare providers can easily access to underpin the clinical decision making process. Technology plays an important role to facilitate access to the information. Gradually, medical professionals around the world are becoming more aware of possibilities to apply technology resources to make more dynamic decisions in caring for clients/patients.

The initial developments in nursing informatics were fragmentary and generally restricted to automating existing functions or activities such as automated charting of nurses' notes, automated personnel time assignment and the gathering of epidemiological and administrative statistics [1]. However, the growing demands work as a stimulating power to the development of broad applications to support nursing practice.

In the 1980s and 1990s, computer and information technology became an integrated part of nursing. Several professional organizations around the world valued the area of expertise and nursing informatics becoming a specialty. Currently, nurses recognize computers as an essential instrument in improving nursing documentation, facilitating analysis, researching and evaluating delivered care in order to enhance the quality of patient care and the maturity of the nursing profession. Nurses form the largest group of health care professionals in any setting hav- 
ing a health information system, whether manual or computerized. For that reason, when providing patient care, nurses make use of an information system more often than any other group of health care professionals. Consequently nurses must play increasing roles in developing, selecting, implementing and evaluating health information systems. A variety of patient care systems are managed by nurses who are introduced to this link during their training. This management knowledge enhances them in embracing information networks and communication skills together with an ability to work both in practice and administrative positions in a variety of clinical and community settings.

Various definitions have been published, based on the development and applications of the area. Nursing Informatics, as originally defined, referred to the use of information technologies in relation to those functions within the purview of nursing, which are carried out by nurses when performing their duties. Hence, any use of information technologies by nurses in relation to the care of patients, the administration of health care facilities or the educational preparation of individuals to practice the discipline is considered nursing informatics [2].

In 1989, Graves and Corcoran [3] published a classical definition that has been used for several years: "nursing informatics is a combination of computer science, information science and nursing science designed to assist in the management and processing of nursing data, information and knowledge to support the practice of nursing and the delivery of nursing care."

The International Medical Informatics Association, Nursing Informatics Special Interest Group (IMIA NI SIG) defines nursing informatics as the "integration of nursing, its information, and information management with information processing and communication technologies to support the health of people world wide." (http://www. imiani.org).

The practice of nursing informatics is broad, ranging from clinical care settings to management of health services, health problems, research, training and education. In this milieu, applications such as decision support systems, quality management, clinical guidelines and telenursing applications are addressed to promote evidence, patient safety and best practice for nursing care delivery.

\section{Discussion}

Nursing informatics is not just about the use of computers in nursing care. Technological tools represent one aspect to be considered; however, the science is the foundation of this investment. Nurses must continue to ensure that information technology remains an integral part of professional practice and that computers are just an instrument with which to acquire the information required. The information we share, we record and we analyze is the main aspect of nursing informatics and it will support the evaluation of our contribution to healthcare services. It also allows us to develop new models that could better utilize resources to improve quality of care and professional performance [4].

As technology continues to evolve, nursing practice methods will also need to adapt in order to take advantage of automation applications. One major effort in nursing informatics during the last three decades of development has been to specify clinical information systems requirements to fulfill nurses' needs for information processing to support practice, education, research and management. To be useful, those systems must capture and process health and health-related data with a broad mix and diversity/scope and a high level of details.

A clinical information system consists of people, information, procedures, hardware and software. Working together all these components can accomplish a set of specific functions to integrate and maximize the benefits of human and technology capabilities. However, to make it happen, existing work flows, routines and practices need to be reviewed and changed. In addition, developers and nurses involved with development and implementation of clinical systems must understand nursing information needs to provide resources that satisfy expectations of the final users in order to enhance practice. It is also wise to consider that implementation of information systems in care settings can produce many predictable and unpredictable impacts in the work environment.

Besides many initiatives in the field, it can still be observed that the use of the computer as an instrument to support nurses' activities in taking care of patients requires more investment in human and material resources. Clinical Systems based on the Nursing Process are not as common as they should be when we consider nurses are the largest group in the healthcare professional mix. The inclusion of nursing elements of practice in the electronic patient record is a nurse responsibility. They need to be involved with the programmers, vendors and developers to drive the nursing process within the profession. Taking care of patients is what nurses know how to do. Therefore, it is essential to ensure that all information required to perform nursing care is present within the computer systems. A nursing care system can be defined as software that automates the nursing 
process in all phases, supporting and facilitating nursing documentation. Since the system development should include means to manage data, nursing care systems including both clinical and administrative functions must integrate. The major purpose of an automated nursing information system is to support nursing activities that can be classified in both interdependent and dependent functions. Interdependent functions include all activities originating from or pertaining to other departments and disciplines such as implementation of medical orders, documentation of admission transfer and discharge data, communication with other departments and disciplines. Independent functions include all activities involved in applying the nursing process.

In clinical settings, one of the most frequent requests from nurses is decision support systems at the point of care. Decision making is an implicit process and occurs in several contexts in the nursing practices. In general aspects, decision support systems are computer systems developed to support some aspects of human decision-making process in order to improve performance and outcomes.

Various systems have been developed and deployed. In the last five years, the major interest is about resources to assure patient safety, which can be considered as one of the most important aspect of healthcare. Nothing is more contrary to the healthcare care philosophy and mission than causing injury or harm to the individuals who search for care [5].

To support nursing administrative tasks, there are systems that can predict staffing, requirements, patients' nursing care needs based on the diagnoses and forecast the nursing unit budget, access patient satisfaction, measure quality and other factions. Major applications that nursing administrators use are: quality assurance, personnel files, communication network, budgeting and payrolls, census, regulatory reporting, forecasting and planning, claims processing and reimbursement, risk pooling, costing nursing care, case-mix and consumer surveys.

Clinical and critical guidelines are also being developed using several different methods for different purposes. Frequently recognized as clinical pathways or care maps, they are based on patient diagnosis, providing a standard evidence-based, time-driven plan of care with predefined interventions to be performed and outcomes to be achieved in targeted time frames [6].

In addition, advances in computer technology and communication have provided consumers with access to a greatly increased amount of information. It is mandatory to mention the power of the Internet that can be used to develop distance learning programs. The Internet offers several online protocols that can support the transmission of information, including electronic mail (e-mail), file transfer protocol (FTP) and the Word Wide Web (WWW) singly and collectively allowing access to resources from anywhere around the world.

Distance learning can be used to train large numbers of nurses in different geographic locations and work settings. To take advantage of the available resources in information technology, nurses should have a minimum of computer-based education established in their educational curriculum. Nurses with little or no previous experience in data standards and computer technology should receive basic training in the use of computer-supported nursing information systems or computer applications in nursing practice.

Telenursing is a way of delivering nursing and health care to individuals, groups and communities. The telecom- munication resources can be used to support different activities such as monitoring medications, fluids and feedings, elaborating physiological examination, providing instruction and helping patients to understand medical procedures, reducing distress and doubts to promote treatment adherence.

Sharing and communicating information is essential in making decisions and delivering care. Exchanging information requires the communicating parties to agree on a communicating channel, an exchange protocol and a common language. The language includes an alphabet, words, phrases, and symbols that express and assign meaning, understood by all users [7].

Computerization has pushed nurses to adapt protocols to document and communicate patient care. The use of common language becomes more critical, and even more demanding to accomplish, in the computerization era. The importance of using standard terminology to describe nursing care is recognized by nurses to facilitate data retrieval and analysis. Efforts have been made in this area and by now different clinical vocabularies are available. However, building a vocabulary that standardizes the clinical nomenclature for use in clinical practice which fulfills all requirements is a continuous challenge and opportunity to the advance of nursing science, building real evidence from practice to enhance care.

\section{International Collaboration}

The focus of IMIA-NI is to foster collaboration among nurses and others who are interested in Nursing Informatics to facilitate development in the field. The aim is to share knowledge, experience and ideas with nurses and healthcare providers worldwide about 
the practice of Nursing Informatics and the benefits of enhanced information management. Specific objectives are identified in the IMIA-NI Strategic Plan [8]. Challenges currently being addressed which lead directly towards the enhancing of care processes in practice and management include:

- Developing the electronic health record that both uses and informs nursing knowledge and is integral to the professional practice of all nurses.

- Developing clinical data standards to enable the development and communication of nursing knowledge and allow the comparison of what nurses do and the results achieved.

- Achieving access to reliable decision support and evidence based care options for clinical care in whatever location the patient receives care, treatment or advice.

- Developing clinical information systems that support the patient journey rather than a specific staff group, while recognising addressing information gaps for nurses.

\section{Conclusions}

At a time when the tendency is toward a computer-based patient record, nurses must be able to identify what kind of nursing data and information will construct useable and useful nursing knowledge. Nurses cannot stand by and wait for vendors and developers to adapt technology to nursing practice.

It is necessary to identify what kind of technological resources can be used to improve nurses' performance. There are many options for each area of application and the selection must be done carefully and fully when considering the enhancement for organizational, technological and local requirements.

\section{Acknowledgement \\ Dr. Heimar Marin acknowledges the support by grant 5D43TW007015-02 from Fogarty International Center and CNPq 305391/2006-2; 201524/2007-4}

\section{References}

1. Hannah KJ, Ball MJ, Edwards MJA, editors. Introduction to Nursing Informatics. Third edition. USA:
Springer; 2006.

2. Hannah KJ. Current trends in nursing informatics: implications for curriculum planning. In: Hannah KJ, Guillemin EJ, Conklin, editors. Nursing uses of computers and information science, Proceedings of the International Symposium on Nursing Uses of Computers and Information Science (Calgary, Canada). Amsterdam: Elsevier, 1985.

3. Graves JR, Corcoran S. The study of nursing informatics. Image 1989; 21(4):227-31.

4 Marin HF, Marques EP. Nursing Informatics: learning from the past to build a new future. Rev Bras Enferm 2005, 58(2):143-6.

5. Marin HF. Improving Patient Safety with technology. Editorial. Int J Med Inform 2004;73:543-6.

6. Taylor S, Farrell A. Vendor applications. In: Saba VK, McCormick KA. Essentials of Nursing Informatics. Fourth edition. McGraw-Hill: USA; 2006. p.423-41.

7. Marin HF, Rodrigues RJ, Delaney C, Nielsen GH, Yan J. Building Standard Based Nursing Information System. Pan American Health Organization: Washington, DC; 2001.

8. IMIA-NI Strategic Plan - Towards IMIA-NI 2015. Ratified August 2007. Available from: http:// f a y r e o r g / i m i a n i / index.php?option=com_docman\&task=cat_view\&gid $=16 \&$ Itemid $=30$

Correspondence to:

Heimar F. Marin

Rua Dep. Bady Bassit 440

05517-050 São Paulo SP. Brazil

E-mail: hfmarin@attglobal.net or heimar@denf.epm.br 